\title{
Enabling $a b$ initio Hessian and frequency calculations of large molecules
}

\author{
Anuja P. Rahalkar, V. Ganesh, ${ }^{a)}$ and Shridhar R. Gadre ${ }^{a), b)}$ \\ Department of Chemistry, University of Pune, Pune 411007, India
}

(Received 28 April 2008; accepted 14 August 2008; published online 15 December 2008)

\begin{abstract}
A linear scaling method, termed as cardinality guided molecular tailoring approach, is applied for the estimation of the Hessian matrix and frequency calculations of spatially extended molecules. The method is put to test on a number of molecular systems largely employing the Hartree-Fock and density functional theory for a variety of basis sets. To demonstrate its ability for correlated methods, we have also performed a few test calculations at the Møller-Plesset second order perturbation theory. A comparison of central processing unit and memory requirements for medium-sized systems with those for the corresponding full ab initio computation reveals substantial gains with negligible loss of accuracy. The technique is further employed for a set of larger molecules, Hessian and frequency calculations of which are not possible on commonly available personal-computer-type hardware. (C) 2008 American Institute of Physics.

[DOI: $10.1063 / 1.2978387]$
\end{abstract}

\section{INTRODUCTION}

The Hessian matrix, comprising of second order partial derivatives of the molecular energy with respect to nuclear coordinates, is one of the most commonly used entity for characterizing the potential energy surfaces of chemical systems and reactions. This can also be used for effecting speedier geometry optimization of molecules. Further, it is essential for computing the vibrational frequencies of a molecule and is also crucial for confirming the local minima or identifying the transition state. However, for ab initio quantum chemical methods, computation of the Hessian matrix is currently an expensive proposition in terms of central processing unit (CPU) and memory requirements. For instance, evaluation of analytic Hessian for Hartree-Fock (HF) method formally scales ${ }^{1,2}$ as $O\left(N^{4}\right)$ for a system represented using $N$ basis functions. When the Hessian is computed numerically, as in the case of density functional theory (DFT), at least $3 M+1$ energy and gradient evaluations are necessary for a system containing $M$ atoms.

To surmount this scalability issue, computational chemists have developed a variety of means and tools for fast evaluation of Hessian and vibrational frequencies at the $a b$ initio level of theory. For instance, Deglmann et al. ${ }^{3}$ proposed various schemes for evaluating frequencies of large molecules using DFT and tested it on several linear molecules and aromatic sheets. Key features of their work involve efficient numerical quadrature, integral prescreening based on rigorous estimates, and exploitation of point group symmetry of the molecule under study. Benchmark results in this work ${ }^{3}$ show that molecules with up to 100 heavy atoms can be treated on a personal computer (PC). Comparisons with experimental values of infra-red (IR) shifts are also made. These techniques are available in the later versions of

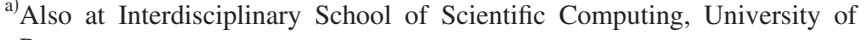
Pune.

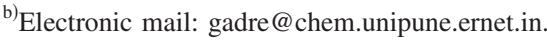

the TURBOMOLE (Ref. 4) package. Another package, ${ }^{5}$ Q-CHEM, incorporates a scalable Hessian evaluation code and has been shown to function well for medium-sized systems containing less than 100 atoms.

A recent paper by Alexeev et al. ${ }^{1}$ discusses a new parallel algorithm for Hessian calculation and has been shown to scale well over a large number of processors for systems such as luciferin and capreomycin. The reported Hessian calculations in all the cases were at the HF level of theory. Their new distributed data analytic method is able to scale over large numbers of processors quite efficiently. Using an advanced HP IA64 based supercomputer interconnected by a very high speed network, the largest of their presented test cases, capreomycin with 778 basis functions, took about 75 min on 256 processors for the Hessian and frequency calculation at the HF/6-31G(d) level of theory. Izmaylov and Scuseria $^{6}$ also recently proposed a method for efficient evaluation of analytical vibrational frequencies in the HF and DFT frameworks for periodic systems. They also applied it for evaluating the vibrational frequencies of various periodic nonconducting systems such as boron nitride sheet and bulk diamond, mostly employing the 6-31G $(d, p)$ basis set. However, the methods presented in this paper ${ }^{6}$ are specifically meant for solids and periodic systems and thus employ periodic boundary conditions.

Neugebauer et $\mathrm{al}^{7}{ }^{7}$ developed methods of approximations in calculations of IR and Raman frequencies and applied their method on buckministerfullerene at various basis sets. Their study is probably the first computational study on IR and Raman spectra of $\mathrm{C}_{60}$ molecule at varying basis sets using numerical Hessian derived from analytical gradients. In a recent study, Pathak and Rastogi ${ }^{8}$ were able to compute IR frequencies for a variety of polycyclic aromatic hydrocarbons (PAHs) at the B3LYP/4-31G level of theory and compared their results with the corresponding experimental ones. All of their calculations were performed on high performance machines using standard GAMESS (Ref. 9) package. 
One of the largest of the calculations that they performed were on PAH $\mathrm{C}_{96} \mathrm{H}_{24}$ at the B3LYP/4-31G level of theory with 912 basis functions. Although the number of basis functions employed is indeed large, the authors' use of symmetry, prevalent in the case of PAHs, considerably reduces the required computational cost.

Besley and Metcalf, ${ }^{10}$ in a recent interesting study, demonstrated the computation of frequencies from partial Hessian for amide I band of a few polypeptides and proteins at the HF and DFT levels of theory. Their method includes the effects of only carbon, oxygen, and nitrogen present on the backbone chain of amino acids. This approximation basically exploits the nature of the amide I band, which is largely localized on carbonyl groups of the backbone amide residues. The mean absolute error that they observed for a set of model polypeptides was of the order of $15 \mathrm{~cm}^{-1}$ at the HF/ STO-3G level of theory. For the model test cases using their method, a time of $10 \%-15 \%$ of the calculation of the full Hessian was observed, which certainly is a large gain in terms of computational requirements, albeit with lesser gains in terms of memory requirements. This method too is integrated with the Q-CHEM package. ${ }^{5}$

To the best of the authors' knowledge, one of the largest frequency calculation using standard $a b$ initio package ${ }^{11}$ is reported in a recent paper by Requena et al. ${ }^{12}$ They reported frequency calculation of $\beta$-carotene and its derivatives, viz., capsanthin and capsorubin, at the B3LYP/6-311G $(d, p)$ level of theory, involving a maximum of 1096 basis functions.

Though parallelization of conventional Hessian calculation procedure does make it easier to handle larger molecules, it in no way addresses the core issue of nonlinear scaling. This practically limits the usability of these methods to systems with typically less than 800 basis functions even with contemporary high performance hardwares. ${ }^{1}$ Baker et al. ${ }^{13}$ however, applied their parallelization scheme for a system of about 1000 basis functions. They reported a timing of about 6 days on an eight processor machine for the case of yohimbine molecule with 1144 basis functions at the DFT level.

The core scalability issue of $a b$ initio codes has been successfully addressed to a large extent by divide-andconquer-type algorithms typically pertaining to one-electron property evaluation, energy evaluation, and geometry optimization. $^{14-27}$ One of these methods, viz., cardinality guided molecular tailoring approach (CG-MTA), is a linear scaling scheme developed in our laboratory for $a b$ initio calculations of large molecules and molecular clusters. Earlier, this approach has been successfully employed for oneelectron property calculation, ${ }^{28-31}$ single point energy and gradient calculation, and geometry optimization ${ }^{16,32,33}$ of a wide variety of molecules using different levels of theory. It may be pointed out that we have inadvertently misquoted in Ref. 32 the origin of the molecular fractionation with conjugated caps approach, which should have been Zhang and co-workers ${ }^{17-20}$ instead of $\mathrm{Li}$ et al. These workers also used $^{17-20}$ linear scaling considerations for one-electron properties and protein-ligand interaction energies.

In the present work, we provide an application of CG-
MTA towards the Hessian and frequency calculations of large systems. These calculations on large molecular systems are typically either very expensive or simply not practically possible using the conventional ab initio methods due to their high CPU and memory requirements. Extensive test results on a number of molecules are presented and compared with actual calculations wherever possible. When actual calculation is not possible because of the hardware limitations, we present a calculation of Hessian and frequency from two or more fragmentation schemes and assess the internal consistency in the results presented. Although the current study is largely restricted to the HF and DFT levels of theory, the technique presented here is applicable to other levels of theory also. This is illustrated with a couple of test cases at the Møller-Plesset second order perturbation theory (MP2). For all the calculations presented here, the parallel CG-MTA scheme is used. ${ }^{32}$ This code is developed in line with various other codes developed earlier ${ }^{34-36}$ in our laboratory.

\section{METHODOLOGY}

As stated earlier, instead of carrying out a full $a b$ initio calculation on a spatially extended large system, CG-MTA breaks it down into a series of calculations on a set of overlapping fragments. A detailed description of the CG-MTA scheme can be found elsewhere, ${ }^{31-33}$ while the most important steps are briefly described here. The method starts with the generation of a set of fragments using the requested parameters, viz., $R$ goodness and maximum allowed size of a fragment. ${ }^{16,31,32}$ Due to the possibility of a multiple fragmentation scheme, effective implementation of CG-MTA requires a quantitative definition of the quality of a given fragmentation scheme. This is achieved by defining minimum $R$ goodness of a fragmentation scheme, details of which can be found elsewhere. ${ }^{16,31-33}$ A better $R$-goodness value generally translates to better quantitative estimates of energy and gradients. Our earlier benchmarks have shown that generally an $R$ goodness of 3-4 $\AA$ produces sufficiently accurate results (up to millihartree accuracy for energy). Once a fragmentation scheme is chosen, the total energy $(E)$ along with its derivatives with respect to the Cartesian coordinates of a supermolecule represented using $k$ fragments can be estimated based on the cardinality expression as given below:

$$
E=\sum E^{f i}-\sum E^{f i \cap f j}+\cdots+(-1)^{k-1} \sum E^{f i \cap f j \cap \cdots \cap f k} .
$$

Here, $E^{f i}$ refers to the energy of fragment $f_{i}, E^{f i \cap f j}$ refers to the energy of the overlap fragment $f_{i} \cap f_{j}$, and so on. Using the above expression for energy, the gradients can now be estimated as

$$
\begin{aligned}
\frac{\partial E}{\partial X_{\mu}}= & \sum \frac{\partial E^{f i}}{\partial X_{\mu}^{f i}}-\sum \frac{\partial E^{f i \cap f j}}{\partial X_{\mu}^{f i \cap f j}} \\
& +\cdots+(-1)^{k-1} \sum \frac{\partial E^{f i \cap f j \cap \cdots \cap f k}}{\partial X_{\mu}^{f i \cap f j \cap \cdots \cap f k}} .
\end{aligned}
$$

Note that in the above equation, $X_{\mu}^{f i}$ and $X_{\mu}^{f i \cap f j}$ refer to the 


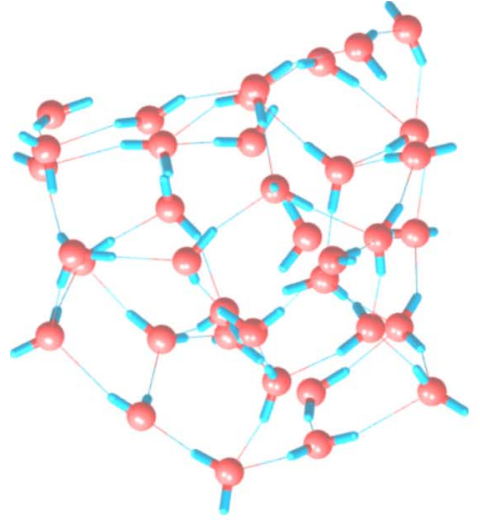

(a)

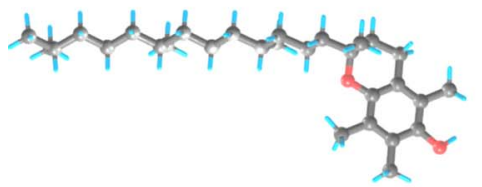

(b)

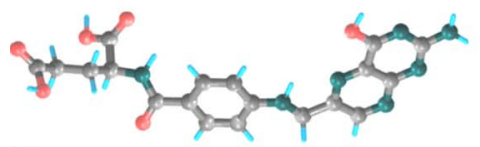

(c)

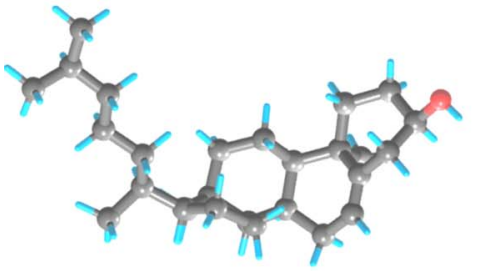

(d)

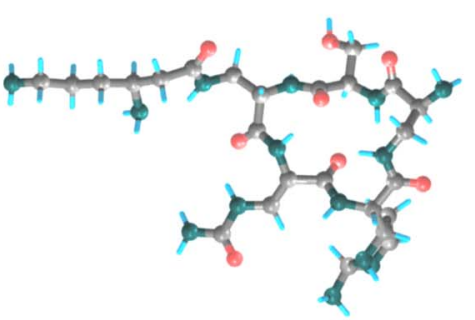

(e)

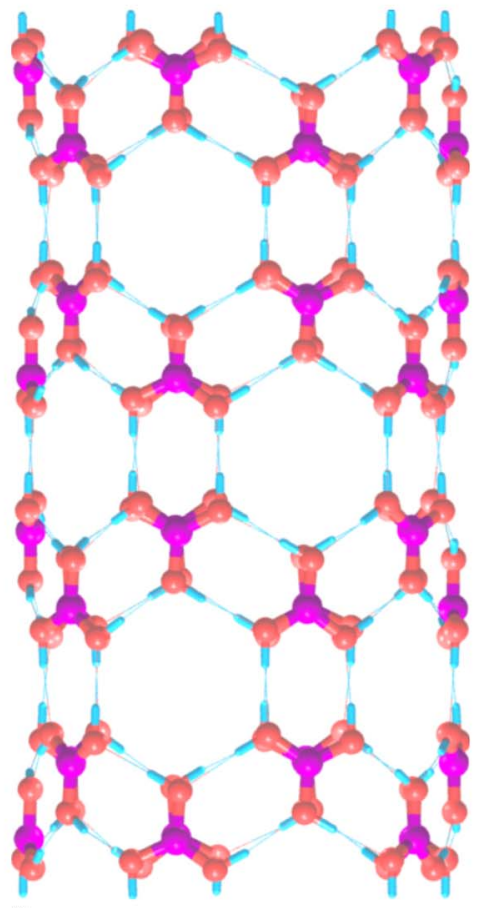

(f)

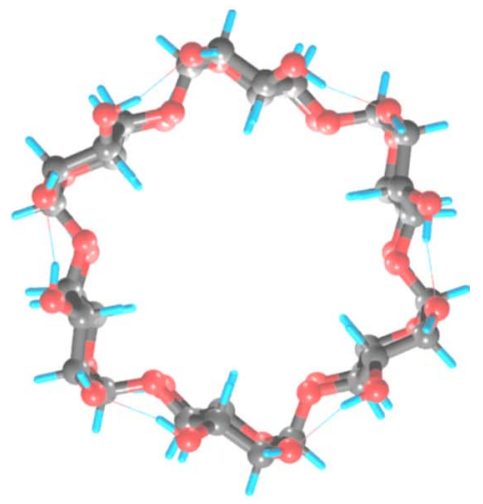

(g)

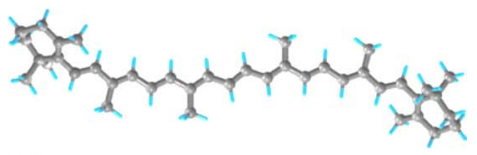

(h)

FIG. 1. (Color online) Geometries of systems put to test for CG-MTA-based Hessian and frequency calculations.

coordinates of atom $\mu$ in the fragment $f_{i}$ and the overlap fragment $f_{i} \cap f_{j}$, etc.

A similar expression is used for computing the $\left(\mu, \mu^{\prime}\right)$ th element of the Hessian matrix:

$$
\begin{aligned}
\mathbf{H}_{\mu \mu^{\prime}}= & \sum \mathbf{H}_{\mu \mu^{\prime}}^{f i}-\sum \mathbf{H}_{\mu \mu^{\prime}}^{f i \cap f j} \\
& +\cdots+(-1)^{k-1} \sum \mathbf{H}_{\mu \mu^{\prime}}^{f i \cap f \cap \cdots \cap f k} .
\end{aligned}
$$

Here, $\mathbf{H}_{\mu \mu^{\prime}}$ refers to the Hessian element corresponding to second derivative of energy with respect to coordinates $X_{\mu}$ and $X_{\mu^{\prime}}$, i.e., $\partial^{2} E / \partial X_{\mu} \partial X_{\mu^{\prime}}$, and so on.

Once the Hessian matrix is set up employing the CGMTA scheme [Eq. (3)], the corresponding vibrational frequencies are extracted by diagonalizing the mass-weighted Hessian matrix $H_{m}$ :

$$
\mathbf{H}_{m}=\mathbf{M}^{-1 / 2} \mathbf{H M}^{\prime-1 / 2},
$$

$$
\mathbf{H}_{m} \mathbf{L}_{k}=\lambda_{k} \mathbf{L}_{k}
$$

Here, $\mathbf{M}$ is the atomic mass array, and $\mathbf{L}_{k}$ and $\lambda_{k}$ are the displacements and frequencies, respectively.

\section{RESULTS AND DISCUSSION}

The majority of the calculations reported here have been performed on a cluster of Intel Core2 Quad processor at $2.4 \mathrm{GHz}$ with 4 Gbyte random access memory (RAM) and 250 Gbytes of disk each. A few of the calculations reported in this work are performed on a cluster of Pentium Dual Core at $2.8 \mathrm{GHz}$ with 2 Gbytes of RAM and 80 Gbytes of local disk. A distributed version of the CG-MTA code ${ }^{16,31,32}$ is used for all the calculations owing to the embarrassingly parallel nature of the algorithm.

Figure 1 displays the structures of test cases investigated in this paper. Table I summarizes the molecular systems, along with the level of theory, basis set used, and number of basis functions involved in the calculation. The spatially extended molecules used as test cases are chemically and biologically diverse in nature and the calculations are performed without enforcing symmetry. The fragmentation details of the systems used as test cases are given in Table I. For all the test cases, a fragmentation scheme with a minimum $R$ goodness of at least $3 \AA$ is employed to ensure the reliability of the calculations performed. ${ }^{16,33}$ The largest fragment size and the scaling factor (see Table I) provide an indirect measure of 
TABLE I. Details of CG-MTA-based Hessian calculation along with the fragmentation schemes. NA and NB represent the numbers of atoms and basis functions of the parent molecule, respectively. NF, AV, LF, and SF denote the number of main fragments, average size of main fragments and number of atoms and basis functions in the largest fragment, and scaling factor, respectively. GP and GN are the pendant and nonpendant $R$-goodness values in $\AA$ (for the definition of pendant and nonpendant $R$-goodness values, see Refs. 16 and 33).

\begin{tabular}{|c|c|c|c|c|c|c|}
\hline System [level/basis] & NA (NB) & NF & $\mathrm{AV}$ & LF & GP, GN & $\mathrm{SF}$ \\
\hline$\left(\mathrm{H}_{2} \mathrm{O}\right)_{37}[\mathrm{HF} / 6-31 \mathrm{G}]$ & $111(481)$ & 8 & 54 & $63(273)$ & $4.1,4.2$ & 4.9 \\
\hline$\alpha$-tocopherol $[\mathrm{HF} / 6-31 \mathrm{G}(d)]$ & $81(565)$ & 6 & 38 & $44(322)$ & $4.5,4.6$ & 2.6 \\
\hline Folic acid (a) [B3LYP/6-31G] & $51(326)$ & 4 & 28 & $33(213)$ & $4.1,4.1$ & 2.0 \\
\hline Folic acid (b) $[\mathrm{B} 3 \mathrm{LYP} / 6-31 \mathrm{G}(d, p)]$ & $51(575)$ & 4 & 28 & $33(375)$ & $4.1,4.1$ & 2.0 \\
\hline$\left(\mathrm{H}_{3} \mathrm{BO}_{3}\right)_{40}[\mathrm{HF} / 6-31++\mathrm{G}(d, p)]$ & $280(3670)$ & & & & & \\
\hline Scheme 1 & & 60 & 23 & $28(376)$ & $3.0,3.0$ & 5.0 \\
\hline Scheme 2 & & 40 & 26 & $28(376)$ & $4.1,4.1$ & 3.8 \\
\hline Cholesterol $[\mathrm{HF} / 6-31 \mathrm{G}(d)]$ & $74(650)$ & 4 & 40 & $42(370)$ & $3.4,3.8$ & 2.0 \\
\hline Capreomycin $[\mathrm{HF} / 6-31+\mathrm{G}(d, p)]$ & $91(1113)$ & 11 & 37 & $43(509)$ & $3.7,3.7$ & 3.9 \\
\hline$\alpha$-cyclodextrin [B3LYP/6-31G $(d)]$ & $126(1110)$ & & & & & \\
\hline Scheme 1 & & 22 & 30 & $36(280)$ & $3.3,3.4$ & 4.3 \\
\hline Scheme 2 & & 12 & 38 & $41(329)$ & $3.3,3.7$ & 3.2 \\
\hline Scheme 3 & & 6 & 55 & $55(461)$ & $3.6,3.7$ & 2.5 \\
\hline$\beta$-carotene (a) $[\mathrm{HF} / 6-31 \mathrm{G}(d)]$ & $96(712)$ & & & & & \\
\hline Scheme 1 & & 10 & 29 & $32(235)$ & $3.9,3.9$ & 2.7 \\
\hline Scheme 2 & & 7 & 32 & $36(254)$ & $4.1,4.2$ & 2.2 \\
\hline$\beta$-carotene (b) $[\mathrm{B} 3 \mathrm{LYP} / 6-31 \mathrm{G}(d)]$ & $96(712)$ & & & & & \\
\hline Scheme 1 & & 5 & 39 & $42(318)$ & $4.2,4.2$ & 1.9 \\
\hline Scheme 2 & & 5 & 36 & $36(280)$ & $4.0,4.0$ & 1.8 \\
\hline
\end{tabular}

the computational cost of a fragmentation scheme. For substantial computational advantage, earlier studies ${ }^{16,33}$ found that this factor should be less than 5 . It should be pointed out here that for folic acid and $\alpha$-tocopherol the largest fragment size is more than half of the parent molecule. This is due to the inherently small size of the parent molecule and hence computational advantage offered by CG-MTA may be small especially if a smaller basis set is employed.

The first three systems in Table I, viz., the cluster of 37 water molecules, $\alpha$-tocopherol, and folic acid, are small but have substantial chemical or biological significance. A fatsoluble antioxidant, $\alpha$-tocopherol is the form of vitamin $\mathrm{E}$ that is preferentially absorbed in humans. Folic acid is a form of water soluble vitamin B. All these systems involve less than 600 basis functions at the specified level of theory, making it possible to run the full frequency calculation (called the "actual calculation" henceforth) using a standard quantum chemical package, GAMESs. ${ }^{9}$ Even in such cases, our method offers notable advantage in terms of computational resources as well as time required for the calculation. For instance, in the case of $\alpha$-tocopherol, the total time taken for this calculation is about $11 \mathrm{~h}$ with CG-MTA as against the staggering $50 \mathrm{~h}$ taken for the actual job on the same hard- ware (see Table II). It is also worth noting that the maximum memory required per node for this job is about 600 Mbytes for CG-MTA while that for the actual job is 1.8 Gbytes. In terms of the accuracy of the Hessian computed using CGMTA as compared to the actual one, the maximum error is $1.8 \times 10^{-4}$ a.u. and root mean square deviation (RMSD) is $1.0 \times 10^{-5}$ a.u. for $\alpha$-tocopherol (see Table III). The other entries from Table III also show that CG-MTA enables the computation of Hessian and vibrational frequencies without significant loss of accuracy.

Important frequencies for $\alpha$-tocopherol are $\mathrm{O}-\mathrm{H}$ stretching at $3604.3 \mathrm{~cm}^{-1}\left(3604.4 \mathrm{~cm}^{-1}\right.$ for CG-MTA) and $\mathrm{C}-\mathrm{O}$ stretching at $1111.8 \mathrm{~cm}^{-1}\left(1111.7 \mathrm{~cm}^{-1}\right.$ for CGMTA). For $\left(\mathrm{H}_{2} \mathrm{O}\right)_{37}$, a wide range of $\mathrm{O}-\mathrm{H}$ stretching frequencies from 3173 to $4106 \mathrm{~cm}^{-1}$ is obtained due to the extensive hydrogen bonding between the water molecules. The important frequencies for folic acid are the $\mathrm{N}-\mathrm{H}$ stretches at 3766.3 and $3621.6 \mathrm{~cm}^{-1}, \mathrm{O}-\mathrm{H}$ stretches in the range of $3715-3762 \mathrm{~cm}^{-1}$, three $\mathrm{C}=\mathrm{O}$ stretches at $1750.8,1835.3$, and $1847.6 \mathrm{~cm}^{-1}$, while $\mathrm{C}-\mathrm{N}$ stretching frequency is observed at $1677.5 \mathrm{~cm}^{-1}$. For all these cases, the difference between CG-MTA and actual frequencies is seen to be within

TABLE II. Comparison of timings of Hessian and frequency calculations for CG-MTA and actual runs. The cores are of Intel Core2 Quad at $2.4 \mathrm{GHz}$.

\begin{tabular}{lccccc}
\hline \hline System & Level/basis & NB & No. of cores & Actual & CG-MTA \\
\hline$\left(\mathrm{H}_{2} \mathrm{O}\right)_{37}$ & $\mathrm{HF} / 6-31 \mathrm{G}$ & 481 & 16 & 1180 & 172 \\
$\alpha$-tocopherol $^{\mathrm{a}}$ & $\mathrm{HF} / 6-31 \mathrm{G}(d)$ & 565 & 6 & 3024 & 666 \\
Folic acid (a) $_{\text {Folic acid (b) }}$ & $\mathrm{B} 3 \mathrm{LYP} / 6-31 \mathrm{G}$ & 326 & 16 & 450 & 270 \\
\hline
\end{tabular}

The cores are of Pentium Dual core at $2.8 \mathrm{GHz}$. 
TABLE III. Accuracy estimates of the Hessian matrix (in a.u.) and vibrational frequencies of the test molecules. (Comparisons only for frequencies greater than $50 \mathrm{~cm}^{-1}$ are reported. The errors in frequencies below $50 \mathrm{~cm}^{-1}$ are also small; however, the percentage error gets inflated and hence these frequencies are not included.)

\begin{tabular}{|c|c|c|c|c|}
\hline \multirow[b]{2}{*}{ System } & \multicolumn{2}{|c|}{ Hessian matrix } & \multicolumn{2}{|c|}{ Frequency $\left(\mathrm{cm}^{-1}\right)$} \\
\hline & Max. error $\left(\times 10^{3}\right)$ & RMSD & $\%$ Max. error (value) & RMSD \\
\hline$\left(\mathrm{H}_{2} \mathrm{O}\right)_{37}$ & 2.91 & 0.07 & $0.48(3188.9)$ & 0.028 \\
\hline$\alpha$-tocopherol & 0.18 & 0.01 & $0.01(243.6)$ & 0.009 \\
\hline Folic acid (a) & 3.24 & 0.18 & $0.47(1081.3)$ & 0.025 \\
\hline Folic acid (b) & 3.24 & 0.18 & $0.32(1508.9)$ & 0.036 \\
\hline$\left(\mathrm{H}_{3} \mathrm{BO}_{3}\right)_{40}{ }^{\mathrm{a}}$ & 0.24 & 0.01 & $0.05(842.5)$ & 0.010 \\
\hline$\alpha$-cyclodextrin ${ }^{\mathrm{b}}$ & 2.65 & 0.12 & $0.29(1105.8)$ & 0.049 \\
\hline$\alpha$-cyclodextrin ${ }^{\mathrm{b}}$ & 1.54 & 0.06 & $0.22(1105.8)$ & 0.012 \\
\hline$\beta$-carotene (a) & 0.94 & 0.00 & $0.07(1777.1)$ & 0.003 \\
\hline$\beta$-carotene (b) & 0.51 & 0.00 & $0.71(197.7)$ & 0.008 \\
\hline
\end{tabular}

${ }^{\mathrm{a}} \mathrm{For}\left(\mathrm{H}_{3} \mathrm{BO}_{3}\right)_{40}$, a comparison of schemes 1 and 2 is reported.

${ }^{\mathrm{b}}$ For $\alpha$-cyclodextrin, comparisons of schemes 1 and 3 (first row) and schemes 2 and 3 (second row) are reported, scheme 3 being the best one.

$1 \mathrm{~cm}^{-1}$. In general, when the complete range of frequencies is compared, the maximum deviation is less than $0.5 \%$ (see Table III).

We next present a few larger test cases for which the actual Hessian and frequency calculations cannot be readily performed on the available hardware. Out of these cases, cholesterol, capreomycin, and $\left(\mathrm{H}_{3} \mathrm{BO}_{3}\right)_{40}$ (a nanotube of 40 orthoboric acid units involving extensively hydrogen bonded network) are treated at the HF level, while $\alpha$-cyclodextrin is treated at the DFT level of theory, employing the basis sets specified in Table I. CG-MTA-optimized geometry of cholesterol is subjected to frequency analysis. Chemically, cholesterol is a combination of steroid and alcohol and plays an important role in the cell membrane of animal tissues. Frequency corresponding to the $\mathrm{O}-\mathrm{H}$ stretch is found at $4179.0 \mathrm{~cm}^{-1}$. Similarly, for capreomycin, a scheme with 11 main fragments is used for CG-MTA geometry optimization. Capreomycin is a peptide antibiotic and is used in combination with other antibiotics for treating tuberculosis. The characteristic frequencies of $\mathrm{C}=\mathrm{O}$ and $\mathrm{N}-\mathrm{H}$ in peptide linkages are obtained in ranges of 1885.6-1950.1 and $3782.2-3927.4 \mathrm{~cm}^{-1}$, respectively, while the $\mathrm{O}-\mathrm{H}$ stretch is obtained at $4197.4 \mathrm{~cm}^{-1}$. The frequency analysis of both the cases confirms the local minimum structure with all the real frequencies.

For $\left(\mathrm{H}_{3} \mathrm{BO}_{3}\right)_{40}$, a CG-MTA based optimized geometry ${ }^{37}$ is taken for frequency analysis. This is carried out using two different fragmentation schemes to appraise the internal consistency of the CG-MTA results. These schemes have $R$-goodness values of 3 and $4.1 \AA$, respectively. The results (in terms of the Hessian and IR frequencies) obtained from both the schemes are found to be in excellent agreement (see Table III) with each other. The frequencies from both the schemes are real and match to within $0.5 \mathrm{~cm}^{-1}$. The time taken by scheme 1 is $25 \mathrm{~h}$ on five dual core machines with 2 Gbyte RAM each, while that for scheme 2 is $12 \mathrm{~h}$ on 13 nodes of the same configuration. It is worth noting here that the number of basis functions involved in these calculations is 3760 , for which actual calculations cannot at all be performed on any commonly available hardware resources. For
$\left(\mathrm{H}_{3} \mathrm{BO}_{3}\right)_{40}$, frequencies within the range of $3970-4190 \mathrm{~cm}^{-1}$ correspond to $\mathrm{O}-\mathrm{H}$ stretches, while those in the range of $1120-1600 \mathrm{~cm}^{-1}$ are due to $\mathrm{B}-\mathrm{O}$ stretches.

Within DFT, the Hessian evaluation is generally done numerically. Hence, even if one wants to use a less accurate two-point formula, it is necessary to evaluate energy and gradients at $3 N_{a}+1$ steps, where $N_{a}$ is the number of atoms in the system. Therefore, the conventional computation of Hessian at the DFT level for a large molecular system consisting of more than 100 atoms is very expensive and time consuming. However, within CG-MTA, the calculations are performed on individual fragments (say, $n_{i}$ atoms) in which, being much smaller than the original molecule ( $N_{a}$ atoms), the number of steps drastically decrease since $3 n_{i}+1 \ll 3 N_{a}$ +1 . Moreover, the fragments being smaller, the effort required for energy-gradient evaluation at each step is also much less than that for the whole molecule.

A cyclic oligosacccharide composed of six glucose units, viz., $\alpha$-cyclodextrin, is chosen as the next test case at the B3LYP/6-31G $(d)$ level. For CG-MTA-based frequency analysis, $\alpha$-cyclodextrin is fragmented with three different schemes. Of these schemes, scheme 3 is the best one with a minimum $R$ goodness of $3.6 \AA$ as both the other schemes have minimum $R$ goodness of $3.3 \AA$. Comparisons of the results obtained from schemes 1 and 2 with those from scheme 3 are displayed in Table III, which evidently bring out self-consistency between the results obtained by the method. The $\mathrm{O}-\mathrm{H}$ stretching frequencies are obtained in the range of $3562-3745 \mathrm{~cm}^{-1}$, while $\mathrm{C}-\mathrm{O}$ stretching frequencies are in the range of $1108-1111 \mathrm{~cm}^{-1}$ from all the three schemes.

Finally, to probe the applicability of CG-MTA for Hessian calculation at the MP2 level, a couple of small test cases, viz., folic acid and $\beta$-carotene, are initially subjected to CG-MTA geometry optimization at the MP2/STO-3G level of theory. These optimized geometries are then used for Hessian and frequency calculation. Folic acid has 179 basis functions for the STO-3G basis, allowing calculations to be performed both by CG-MTA and the conventional method. The actual Hessian and frequency calculation took $44.5 \mathrm{~h}$ on 
a Pentium IV at $2.8 \mathrm{GHz}$ with 1 Gbyte of RAM, while CGMTA calculation took $15.4 \mathrm{~h}$ on the same hardware. The maximum memory required for actual calculation is 450 Mbytes while that for CG-MTA is merely 85 Mbytes. In terms of the accuracy of the Hessian matrix, the maximum error is $1.3 \times 10^{-5}$ and all the frequencies (in $\mathrm{cm}^{-1}$ ) are correct to all places before the decimal point. The above geometry is further optimized at the MP2/3-21G level of theory with CG-MTA and then subjected to frequency calculation. This calculation involved 326 basis functions and hence an actual calculation is not possible with the above mentioned hardware. In order to prove the stability of the method at the MP2 level, two different fragmentation schemes with minimum goodness values of 4.0 and $4.8 \AA$ are used for this calculation. The maximum deviation in the Hessian elements is 0.04966 and RMSD is $6.9 \times 10^{-4}$ a.u. The RMSD in the computed frequencies from the two schemes is $0.011 \mathrm{~cm}^{-1}$; however, the \% maximum error is 1.22 , which is on the higher side as compared to other test results. This could be due to the inability of the current fragmentation schemes to appropriately capture some of the correlation effects. Hence, it is expected that better quality fragments will produce more accurate results.

The other test case of $\beta$-carotene consists of 256 basis functions at the MP2/STO-3G level of theory. In GAMESS, Hessian is numerically calculated at the MP2 level, so actual calculation for $\beta$-carotene requires 289 steps of energy and gradient evaluation $\left(3 N_{a}+1\right.$ steps, with $N_{a}=$ number of atoms in the system). One such GAMESS-based step takes about $30 \mathrm{~min}$ on 16 cores. Hence the complete calculation is expected to run for about 1 week. The CG-MTA-based calculations are performed using two different fragmentation schemes with $R$-goodness values of 3.9 and $4.1 \AA$, respectively. The time advantage offered by CG-MTA is worth pointing out here. CG-MTA based calculations using both of these schemes are completed within 50 min on 20 cores. The results from both the schemes are consistent with each other and the maximum error in the frequencies obtained using these two schemes is $2.7 \mathrm{~cm}^{-1}$. These results bring out the applicability of CG-MTA for higher correlated methods, which is being pursued further in our laboratory.

\section{CONCLUDING REMARKS}

The present work has explored the use of CG-MTA for Hessian and frequency calculation of large molecular systems. For the test cases we have presented, the CG-MTA based Hessian is observed to be highly accurate when compared to its actual counterpart. The typical maximum error in any Hessian element is of the order of only $10^{-3}$ a.u. All the frequencies derived from the CG-MTA based Hessian are generally found to be correct up to $0.5 \%$. In terms of memory and CPU requirements, a substantial savings is seen while comparing a CG-MTA calculation with the conventional one.

The test cases presented in this work clearly show that CG-MTA indeed enables calculations of the Hessian matrix and vibrational frequencies that are otherwise not possible on contemporary PC-based hardware without significant loss of accuracy. The Hessian and frequency calculations, without any symmetry constraints, for a large molecule such as $\left(\mathrm{H}_{3} \mathrm{BO}_{3}\right)_{40}$ with 3670 basis functions, to the best of our knowledge, is the largest reported one. In the future, this technique could be extended for application of Hessian for geometry optimization or locating transition states, etc. Although the current study is mainly restricted to the HF and DFT levels of theory, we have also shown its applicability to correlated theories (MP2). Further studies with the MP2 level of theory are currently under way in our laboratory.

Note added in proof. A related work on geometry optimization and vibrational spectra of large molecules by Hua et $a l^{38}$ appeared after the present manuscript was accepted for publication. This work has discussed the vibrational frequencies along with IR and raman intensities for 12-mer of glycine and 28-mer of water. A more detailed comparison of our work with that of Hua et al. will be made in a forthcoming publication. ${ }^{39}$

\section{ACKNOWLEDGMENTS}

Financial support from the Center for Development of Advanced Computing (C-DAC), Pune, Naval Research Board (NRB), New Delhi, and Council of Scientific and Industrial Research (CSIR), New Delhi is gratefully acknowledged. The authors are also thankful to Dr. Michael Schmidt from Iowa State University for useful suggestions.

${ }^{1}$ Y. Alexeev, M. W. Schmidt, T. L. Windus, and M. S. Gordon, J. Comput. Chem. 28, 1685 (2007).

${ }^{2}$ H. Horn, H. Weiss, M. Ehrig, and R. Ahlrichs, J. Comput. Chem. 9, 1058 (1991).

${ }^{3}$ P. Deglmann, F. Furche, and R. Ahlrichs, Chem. Phys. Lett. 352, 511 (2002).

${ }^{4}$ R. Ahlrichs, M. Bar, M. Haser, H. Horn, and C. Kölmel, Chem. Phys. Lett. 162, 165 (1989).

${ }^{5}$ Y. Shao, L. F. Molnar, Y. Jung, J. Kussmann, C. Ochsenfeld, S. T. Brown, A. T. B. Gilbert, L. V. Slipchenko, S. V. Levchenko, D. P. O'Neill, R. A. DiStasio, Jr., R. C. Lochan, T. Wang, G. J. O. Beran, N. A. Besley, J. M. Herbert, C. Y. Lin, T. V. Voorhis, S. H. Chien, A. Sodt, R. P. Steele, V. A. Rassolov, P. E. Maslen, P. P. Korambath, R. D. Adamson, B. Austin, J. Baker, E. F. C. Byrd, H. Daschel, R. J. Doerksen, A. Dreuw, B. D. Dunietz, A. D. Dutoi, T. R. Furlani, S. R. Gwaltney, A. Heyden, S. Hirata, C. P. Hsu, G. Kedziora, R. Z. Khalliulin, P. Klunzinger, A. M. Lee, M. S. Lee, W. Z. Liang, I. Lotan, N. Nair, B. Peters, E. I. Proynov, P. A. Pieniazek, Y. M. Rhee, J. Ritchie, E. Rosta, C. D. Sherrill, A. C. Simmonett, J. E. Subotnik, H. L. Woodcock III, W. Zhang, A. T. Bell, and A. K. Chakraborty, Phys. Chem. Chem. Phys. 8, 3172 (2006).

${ }^{6}$ A. F. Izmaylov and G. E. Scuseria, J. Chem. Phys. 127, 144106 (2007). ${ }^{7}$ J. Neugebauer, M. Reiher, C. Kind, and B. A. Hess, J. Comput. Chem. 23, 895 (2002).

${ }^{8}$ A. Pathak and S. Rastogi, Spectrochim. Acta, Part A 67, 898 (2007).

${ }^{9}$ The Gamess package, M. W. Schmidt, K. K. Baldridge, J. A Boatz, S. T. Elbert, M. S Gordon, J. H Jensen, S. Koseki, N. Matsunaga, K. A. Nguyen, S. J. Su, T. L. Windus, and M. Dupuis, J. Comput. Chem. 14, 1347 (1993).

${ }^{10}$ N. A. Besley and K. A. Metcalf, J. Chem. Phys. 126, 035101 (2007).

${ }^{11}$ M. J. Frisch, G. W. Trucks, H. B. Schlegel et al., GAUSSIAN 03, Revision C.02, Gaussian, Inc., Wallingford, CT, 2004.

${ }^{12}$ A. Requena, J. P. Ceron-Carrasco, A. Bastida, J. Zuniga, and B. Miguel, J. Phys. Chem. A 112, 4815 (2008).

${ }^{13}$ J. Baker, K. Wolinski, M. Malagoli, and P. Pulay, Mol. Phys. 102, 2475 (2004).

${ }^{14}$ W. Yang and T. S. Lee, J. Chem. Phys. 103, 5674 (1995).

${ }^{15}$ T. S. Lee, J. P. Lewis, and W. Yang, Comput. Mater. Sci. 12, 1259 (1998).

${ }^{16}$ S. R. Gadre and V. Ganesh, J. Theor. Comput. Chem. 5, 835 (2006).

${ }^{17}$ D. W. Zhang and J. Z. H. Zhang, J. Chem. Phys. 119, 3599 (2003). 
${ }^{18}$ Y. Xiang, D. W. Zhang, and J. Z. H. Zhang, J. Comput. Chem. 25, 1431 (2004).

${ }^{19}$ For a work on one-electron properties using similar equations as Eq. (1) in the present work, see A. M. Gao, D. W. Zhang, J. Z. H. Zhang, and Y. Zhang, Chem. Phys. Lett. 394, 293 (2004).

${ }^{20}$ X. Chen, Y. Zhang, and J. Z. H. Zhang, J. Chem. Phys. 122, 184105 (2005).

${ }^{21}$ K. Fukuzawa, K. Kiraura, M. Uebayasi, K. Nakata, T. Kaminuma, and T. Nakano, J. Comput. Chem. 26, 1 (2005).

${ }^{22}$ S. Li, W. Li, and T. Fang, J. Am. Chem. Soc. 127, 7215 (2005).

${ }^{23}$ V. Deev and M. A. Collins, J. Chem. Phys. 122, 154102 (2005).

${ }^{24}$ V. Deev and M. A. Collins, J. Chem. Phys. 127, 104104 (2006).

${ }^{25}$ M. A. Collins, J. Chem. Phys. 127, 024104 (2007).

${ }^{26}$ D. G. Fedorov, T. Ishida, M. Uebayasi, and K. Kitaura, J. Phys. Chem. A 111, 2722 (2007).

${ }^{27}$ D. G. Fedorov and K. Kitaura, J. Phys. Chem. A 111, 6904 (2007).

${ }^{28}$ S. R. Gadre, R. N. Shirsat, and A. C. Limaye, J. Phys. Chem. 98, 9165 (1994).

${ }^{29}$ K. Babu and S. R. Gadre, J. Comput. Chem. 24, 484 (2003).
${ }^{30}$ K. Babu, V. Ganesh, S. R. Gadre, and N. E. Ghermani, Theor. Chem. Acc. 111, 255 (2004)

${ }^{31}$ S. R. Gadre, K. Babu, and V. Ganesh, Recent Trends in Practice and Theory of Information Technology: Proceedings of NRB Seminar, edited by S. N. Maheshwari (Viva Books, New Delhi, 2005).

${ }^{32}$ V. Ganesh, R. K. Dongare, P. Balanarayan, and S. R. Gadre, J. Chem. Phys. 125, 104109 (2006).

${ }^{33}$ S. R. Gadre, A. P. Rahalkar, and V. Ganesh, IANCAS Bulletin 4, 267 (2006).

${ }^{34}$ S. R. Gadre, S. A. Kulkarni, A. C. Limaye, and R. N. Shirsat, Z. Phys. D: At., Mol. Clusters 18, 357 (1991).

${ }^{35}$ S. R. Gadre, A. C. Limaye, and R. N. Shirsat, J. Comput. Chem. 14, 445 (1993).

${ }^{36}$ S. R. Gadre and A. C. Limaye, J. Chem. Phys. 100, 1303 (1993).

${ }^{37}$ M. Elango, V. Subramanian, A. P. Rahalkar, S. R. Gadre, and N. Sathyamurthy, J. Phys. Chem. A 112, 7699 (2008).

${ }^{38}$ W. Hua, T. Fang, W. Li, J. G. Yu, and S. Li, J. Phys. Chem. A 112, 10864 (2008).

${ }^{39}$ S. R. Gadre, K. V. Jovan Jose, and A. P. Rahalkar, J. Chem. Sci. (to be published). 